\title{
Update: Characteristics of Patients in a National Outbreak of E-cigarette, or Vaping, Product Use-Associated Lung Injuries — United States, October 2019
}

\author{
Erin D. Moritz, $\mathrm{PhD}^{1}$; Lauren B. Zapata, $\mathrm{PhD}^{2}$; Akaki Lekiachvili, $\mathrm{MD}^{2}$; Emily Glidden, $\mathrm{MPH}^{1}$; Francis B. Annor, $\mathrm{PhD}^{3}$; \\ Angela K. Werner, $\mathrm{PhD}^{1}$; Emily N. Ussery, $\mathrm{PhD}^{2}$; Michelle M. Hughes, PhD ${ }^{4}$; Anne Kimball, MD ${ }^{5,6}$; Carla L. DeSisto, PhD ${ }^{2,5}$; \\ Brandon Kenemer, $\mathrm{MPH}^{2}$; Mays Shamout, $\mathrm{MD}^{2,5}$; Macarena C. Garcia, DrPH${ }^{7}$; Sarah Reagan-Steiner, $\mathrm{MD}^{8}$; Emily E. Petersen, $\mathrm{MD}^{2}$; \\ Emily H. Koumans, $\mathrm{MD}^{2}$; Matthew D. Ritchey, DPT ${ }^{2}$; Brian A. King, $\mathrm{PhD}^{2}$; Christopher M. Jones, DrPH $\mathrm{PH}^{3}$ Peter A. Briss, $\mathrm{MD}^{2}$; \\ Lisa Delaney, MS ; Anita Patel, PharmD ${ }^{10}$; Kara D. Polen, MPH ${ }^{4}$; Katie Sives, MPH ${ }^{2}$; Dana Meaney-Delman, MD ${ }^{4}$; Kevin Chatham-Stephens, MD ${ }^{4}$; \\ Lung Injury Response Epidemiology/Surveillance Group
}

On October 28, 2019, this report was posted as an MMWR Early Release on the MMWR website (https://www.cdc.gov/mmwr).

CDC, the Food and Drug Administration, state and local health departments, and other public health and clinical stakeholders are investigating a national outbreak of electroniccigarette (e-cigarette), or vaping, product use-associated lung injury (EVALI) (1). As of October 22, 2019, 49 states, the District of Columbia (DC), and the U.S. Virgin Islands have reported 1,604 cases of EVALI to CDC, including 34 (2.1\%) EVALI-associated deaths in 24 states. Based on data collected as of October 15, 2019, this report updates data on patient characteristics and substances used in e-cigarette, or vaping, products (2) and describes characteristics of EVALI-associated deaths. The median age of EVALI patients who survived was 23 years, and the median age of EVALI patients who died was 45 years. Among 867 (54\%) EVALI patients with available data on use of specific e-cigarette, or vaping, products in the 3 months preceding symptom onset, $86 \%$ reported any use of tetrahydrocannabinol (THC)-containing products, $64 \%$ reported any use of nicotine-containing products, and $52 \%$ reported use of both. Exclusive use of THC-containing products was reported by $34 \%$ of patients and exclusive use of nicotine-containing products by $11 \%$, and for $2 \%$ of patients, no use of either THC- or nicotine-containing products was reported. Among 19 EVALI patients who died and for whom substance use data were available, $84 \%$ reported any use of THC-containing products, including 63\% who reported exclusive use of THC-containing products; 37\% reported any use of nicotine-containing products, including $16 \%$ who reported exclusive use of nicotine-containing products. To date, no single compound or ingredient used in e-cigarette, or vaping, products has emerged as the cause of EVALI, and there might be more than one cause. Because most patients reported using THC-containing products before symptom onset, CDC recommends that persons should not use e-cigarette, or vaping, products that contain THC. In addition, because the specific compound or ingredient causing lung injury is not yet known, and while the investigation continues, persons should consider refraining from the use of all e-cigarette, or vaping, products.

State health departments, the Council of State and Territorial Epidemiologists Vaping Associated Pulmonary Injury
Epidemiology Task Force, and CDC developed and disseminated surveillance case definitions* and data collection tools (i.e., patient interview and medical record abstraction forms) to monitor and track cases beginning in August 2019. Some states are using these tools, whereas others elected to use state-specific tools. States and jurisdictions routinely report the number of confirmed and probable EVALI cases to CDC on a voluntary basis and, when available, include data from medical record abstractions and patient interviews. Proxies (e.g., spouses or parents) were interviewed if patients were too ill or if they had died. Most states and jurisdictions report the number of cases to CDC as case status is determined; however, it can take up to several weeks to complete and submit information from interview and medical record abstraction. This report provides updated data on patient demographic characteristics; substances used in e-cigarette, or vaping, products; and characteristics of EVALI patients who died, based on cases reported to CDC with available interview and medical record abstraction data as of October 15, 2019. The median ages of patients were compared across groups using the Wilcoxon rank-sum test. SAS statistical software (version 9.4; SAS Institute) was used for the analysis.

As of October 22, 2019, 49 states, DC, and the U.S. Virgin Islands had reported 1,604 cases of EVALI to CDC, including 34 (2.1\%) EVALI-associated deaths in 24 states. Among 1,378 patients with confirmed or probable EVALI reported to CDC by October 15, 2019, with available data, 964 (70\%) were male (Table). No cases in pregnant women were reported. Among 1,364 patients with information on age, the median age was 24 years (range $=13-75$ years) and was similar among males (23 years) and females (25 years); 737 (54\%) patients were aged $<25$ years, and $1,081(79 \%)$ were aged $<35$ years. Among 383 EVALI patients with available information on race/ethnicity, $298(78 \%)$ were non-Hispanic white, and $62(16 \%)$ were Hispanic. Among 867 patients with available data on substances used, $749(86 \%)$ reported any use of THC-containing products, and $552(64 \%)$ reported any use of nicotine-containing products in the 3 months preceding symptom onset; 455 patients (52\%) reported use of both

\footnotetext{
*https://www.cdc.gov/tobacco/basic_information/e-cigarettes/assets/2019-LungInjury-Surveillance-Case-Definition-508.pdf.
} 


\section{Summary}

What is already known about this topic?

CDC and partners are investigating the ongoing outbreak of e-cigarette, or vaping, product use-associated lung injury (EVALI) in the United States, the District of Columbia, and one U.S. territory.

What is added by this report?

As of October 22, 2019, a total of 1,604 cases of EVALI, including 34 deaths, were reported to CDC. Based on data collected as of October 15, 2019, use of tetrahydrocannabinol (THC)-containing products in the 3 months preceding symptom onset was reported by $86 \%$ of patients. The median age of EVALI patients who survived was 23 years, and the median age of EVALI patients who died was 45 years.

What are the implications for public health practice?

Most EVALI patients report using THC-containing products before symptom onset. CDC recommends that persons should not use e-cigarette, or vaping, products containing THC. Because the specific compound or ingredient causing EVALI is not known, persons should consider refraining from use of all e-cigarette, or vaping, products.

THC-containing products and nicotine-containing products, 294 (34\%) reported exclusive use of THC-containing products, and $97(11 \%)$ reported exclusive use of nicotinecontaining products. Twenty-one (2\%) patients reported no use of THC- or nicotine-containing products.

Among the 29 EVALI-associated deaths reported to CDC as of October 15, 2019, 59\% (17) were male; the median age was 45 years (range $=17-75$ years) overall (Table), 55 years (range $=17-71$ years) among males, and 43 years (range $=27-75$ years) among females; the age difference between males and females was not statistically significant $(\mathrm{p}=0.5)$. Patients who died were older than patients who survived $(p<0.01)$. Among 19 EVALI patients who died and for whom data on substance use was available, the use of any THC-containing products was reported by patients or proxies for $84 \%$ (16), including 63\% (12) who exclusively used THC-containing products. Use of any nicotine-containing products was reported for 37\% (seven), including 16\% (three) who exclusively used nicotine-containing products. Use of both THC- and nicotine-containing products was reported in four decedents.

\section{Discussion}

Cases of EVALI continue to be reported to CDC as part of this national outbreak. Similar to previous reports at the national and state levels (1-4), most patients reported use of THC-containing products in the 3 months before symptom onset. Patients were predominantly aged $<35$ years,
non-Hispanic white, and male. Patients with EVALI who died were older than patients who survived. Illnesses and deaths occurred across an age spectrum, from adolescents to older adults. Approximately half of cases, and two deaths, occurred in patients aged $<25$ years. Older adults were disproportionately represented among patients who died; only $2 \%$ of cases, but nearly $25 \%$ of deaths, occurred in patients aged $>65$ years. Further, any use of THC-containing products was reported for $86 \%$ of patients who survived and $84 \%$ of patients who died; exclusive use of THC-containing products was reported for $63 \%$ of EVALI patients who died and for 33\% who survived.

Findings from this report, which is the largest analysis of EVALI patients to date, suggest that this outbreak continues to substantially affect young persons, highlighting the need to communicate the dangers of e-cigarette, or vaping, use particularly among youths and young adults. Although 2\% of all EVALI patients were aged $65-75$ years, $24 \%$ of deaths were in this age group; relevant tailored and targeted messaging might also be needed for this age group. Consistent with previously published reports (1-4), the data presented here suggest that THC-containing products are playing an important role in this outbreak. Further, reports from Illinois, Utah, and Wisconsin suggest that patients have typically obtained their THC-containing e-cigarette, or vaping, products through informal sources, such as friends or illicit in-person and online dealers, although local and regional differences in illicit THC supply and production might exist $(3,4)$.

The findings in this report are subject to at least three limitations. First, data on substances used in e-cigarette, or vaping, products were self-reported or reported by proxies and might be subject to recall bias, as well as social desirability bias because nonmedical marijuana is illegal in many states. Therefore, underreporting might have occurred, particularly for patients who died and others whose information was provided by a proxy. Second, data on some variables, such as race/ethnicity, were missing for many patients, and conclusions based on these data might not be generalizable to the entire patient population. Finally, these data might be subject to misclassification of substance use for multiple reasons. Patients likely did not know the content of the e-cigarette, or vaping, products they used, and methods used to collect substance use data varied across states.

To date, no single compound or ingredient has emerged as the cause of EVALI, and there might be more than one cause. Because most patients report using THC-containing products before the onset of symptoms, CDC recommends that persons should not use e-cigarette, or vaping, products that contain THC. Persons should not buy any type of e-cigarette, or vaping, products, particularly those containing THC, off the street and should not modify or add any substances to 
TABLE. Characteristics of patients with electronic cigarette (e-cigarette), or vaping, product use-associated lung injury (EVALI) reported to CDC — United States, August-October 2019*

\begin{tabular}{|c|c|c|c|}
\hline \multirow[b]{2}{*}{ Characteristic } & \multicolumn{3}{|c|}{ No. $/$ Total No. $\left(\%^{\dagger}\right)$} \\
\hline & EVALI patients who survived & EVALI-associated deaths & All EVALI patients \\
\hline \multicolumn{4}{|l|}{ Sex } \\
\hline Male & $947 / 1,349(70)$ & $17 / 29(59)$ & $964 / 1,378(70)$ \\
\hline Female & $402 / 1,349(30)$ & $12 / 29(41)$ & $414 / 1,378(30)$ \\
\hline \multicolumn{4}{|l|}{ Age group (yrs) } \\
\hline $13-17$ & $735 / 1,335(55)^{\S}$ & $2 / 29(7)^{\S}$ & $196 / 1,364(14)$ \\
\hline $18-24$ & & & $541 / 1,364(40)$ \\
\hline $25-34$ & $339 / 1,335(25)$ & $5 / 29(17)$ & $344 / 1,364(25)$ \\
\hline $35-44$ & $165 / 1,335(12)$ & $7 / 29(24)$ & 172/1,364 (13) \\
\hline $45-64$ & 79/1,335 (6) & $8 / 29(28)$ & 87/1,364 (6) \\
\hline $65-75$ & $17 / 1,335(1)$ & $7 / 29(24)$ & 24/1,364 (2) \\
\hline \multicolumn{4}{|l|}{ Median age, yrs (range) } \\
\hline Overall & $23(13-72)$ & $45(17-75)$ & $24(13-75)$ \\
\hline Male & $23(13-68)$ & $55(17-71)$ & $23(13-71)$ \\
\hline Female & $25(13-72)$ & $43(27-75)$ & $25(13-75)$ \\
\hline \multicolumn{4}{|l|}{ Race/Ethnicityף } \\
\hline White & 283/365 (78) & $15 / 18(83)$ & 298/383 (78) \\
\hline Black or African American & $22 / 365(6)^{* *}$ & $1 / 18(6)^{* *}$ & 9/383 (2) \\
\hline American Indian or Alaska Native & & & 4/383(1) \\
\hline Asian, Native Hawaiian, or other Pacific Islander & & & $5 / 383(1)$ \\
\hline Other & & & $5 / 383(1)$ \\
\hline Hispanic & $60 / 365(16)$ & $2 / 18(11)$ & $62 / 383(16)$ \\
\hline \multicolumn{4}{|l|}{ Substances used in e-cigarette, or vaping, products ${ }^{\dagger+, \S \S ~}$} \\
\hline THC-containing products, any use & 733/848 (86) & 16/19 (84) & 749/867 (86) \\
\hline Nicotine-containing products, any use & $545 / 848(64)$ & 7/19(37) & $552 / 867(64)$ \\
\hline Both THC- and nicotine-containing products, any use & $451 / 848(53)$ & 4/19(21) & 455/867 (52) \\
\hline THC-containing products, exclusive use & 282/848 (33) & $12 / 19(63)$ & 294/867 (34) \\
\hline Nicotine-containing products, exclusive use & $94 / 848(11)$ & $3 / 19(16)$ & 97/867 (11) \\
\hline No THC- or nicotine-containing products reported & $21 / 848(2)$ & $0 / 19(0)$ & $21 / 867(2)$ \\
\hline
\end{tabular}

\footnotetext{
Abbreviation: $\mathrm{THC}=$ tetrahydrocannabinol.

* Reported as of October 15, 2019.

t Percentages might not add up to $100 \%$ because of rounding.

$\S$ Data for the 13-17 and 18-24 age groups were combined to protect patient identity.

" Whites; blacks or African Americans; American Indians or Alaska Natives; Asians, Native Hawaiians and other Pacific Islanders; and Others were non-Hispanic. Hispanic persons could be of any race.

** Data for persons in the following race/ethnicity groups were combined to protect patient identity: black or African American; American Indian or Alaska Native, Asian, Native Hawaiian, or other Pacific Islander, and Other.

t+ In the 3 months preceding symptom onset; categories not mutually exclusive.

$\S \S$ Data on both THC- and nicotine-containing product use required to be included.
}

e-cigarette, or vaping, products that are not intended by the manufacturer, including products purchased through retail establishments. In addition, because the specific compound or ingredient causing lung injury is not yet known, and while the investigation continues, persons should consider refraining from use of all e-cigarette, or vaping, products. E-cigarette, or vaping, products should never be used by youths, young adults, or women who are pregnant. Moreover, persons who do not currently use tobacco products should not start using e-cigarette, or vaping, products $(2,5)$.

\section{Acknowledgments}

Sarah Khalidi, Sondra Reese, Alabama Department of Public Health; Appathurai Balamurugan, Allison James, Arkansas Department of Health; Monique Adakai, Emily Carlson, Arizona Department of Health Services; Armando Chevez,
Daniel U. Kwon, California Department of Public Health; Elyse Contreras, Katelyn E. Hall, Colorado Department of Public Health and Environment; Sydney Jones, Connecticut Department of Public Health; Adrienne Sherman, Kenan Zamore, District of Columbia Department of Health; Amanda Bundek, Caroline Judd, Division of Public Health, Delaware Department of Health and Social Services; Heather Rubino, Thomas Troelstrup, Florida Department of Health; Georgia Department of Public Health Lung Injury Response Team; Hawaii Department of Health; Chris Galeazzi, Ben Williamson, Iowa Department of Public Health; Eileen M. Dunne, Kathryn A. Turner, Idaho Division of Public Health; Dawn Nims, Lori Saathoff-Huber, Illinois Department of Public Health; Kathryn Gaub, Sara Hallyburton, Indiana State Department of Health; Amie Cook, Kansas Department of Health and Environment; Kentucky Department for Public Health; Julie Hand, Theresa Sokol, Louisiana Department of Health; Daniel Church, MaryKate Martelon, Massachusetts Department of Public Health; Kenneth A. 
Feder, Clifford S. Mitchell, Maryland Department of Health; Maine Center for Disease Control and Prevention; Rita Seith, Eden V. Wells, Michigan Department of Health and Human Services; Stacy Holzbauer, Terra Wiens, Minnesota Department of Health; Valerie Howard, George Turabelidze, Missouri Department of Health and Senior Services; Paul Byers, Kathryn Taylor, Mississippi State Department of Health; Kim Bailey, RiverStone Health; William Gavin, Gallatin City-County Health Department; Ariel Christensen, Molly N. Hoffman, North Carolina Division of Public Health; Clint Boots, Tracy Miller, Kodi Pinks, North Dakota Department of Health; Matthew Donahue, Tom Safranek, Nebraska Department of Health and Human Services; Suzann Beauregard, Pascal Kalin, New Hampshire Department of Health and Human Services; Lisa McHugh, Stephen Perez, New Jersey Department of Health; Alex Gallegos, Joseph T. Hicks, New Mexico Department of Health; Ashleigh Faulstich, Victoria LeGarde, Melissa Peek-Bullock, Nevada Department of Health and Human Services; Adam Helman, Kristen Navarette, New York State Department of Health; Courtney Dewart, Kirtana Ramadugu, Ohio Department of Health; Claire B. Nguyen, Tracey Wendling, Oklahoma State Department of Health; Amanda Faulkner, Tasha Poissant, Oregon Health Authority; Joann F. Gruber, Laurel Harduar Morano, Pennsylvania Department of Health; Ailis Clyne, Morgan Orr, James Rajotte, Rhode Island Department of Health; Sharon Biggers, Virginie Daguise, Daniel Kilpatrick, South Carolina Department of Health \& Environmental Control;
Joshua L. Clayton, Jonathan Steinberg, South Dakota Department of Health; Julie Shaffner, Kelly Squires, Tennessee Department of Health; Emily Hall, Varun Shetty, Texas Department of State Health Services; Esther M. Ellis, U.S. Virgin Islands Department of Health; Nathaniel Lewis, Utah Department of Health; Jonathan Falk, Lilian Peake, Virginia Department of Health; Vermont Department of Health; Michelle Holshue, Cathy Wasserman, Washington State Department of Health; Staff members, Wisconsin Department of Health Services; Shannon McBee, Christy Reed, West Virginia Department of Health and Human Resources; and Melissa Taylor, Wyoming Department of Health.

Corresponding author: Erin D. Moritz, emoritz@cdc.gov.

\begin{abstract}
${ }^{1}$ National Center for Environmental Health, CDC; ${ }^{2}$ National Center for Chronic Disease Prevention and Health Promotion, CDC; ${ }^{3}$ National Center for Injury Prevention and Control, CDC; ${ }^{4}$ National Center on Birth Defects and Developmental Disabilities, CDC; ${ }^{5}$ Epidemic Intelligence Service, CDC; ${ }^{6}$ National Center for HIV/AIDS, Viral Hepatitis, STD, and TB Prevention, CDC; ${ }^{7}$ Center for Surveillance, Epidemiology, and Laboratory Services, CDC; ${ }^{8}$ National Center for Emerging and Zoonotic Infectious Diseases, CDC; ${ }^{9}$ National Institute for Occupational Safety and Health, CDC; ${ }^{10}$ National Center for Immunization and Respiratory Disease, CDC.
\end{abstract}

All authors have completed and submitted the International Committee of Medical Journal Editors form for disclosure of potential conflicts of interest. No potential conflicts of interest were disclosed.

\section{Lung Injury Response Epidemiology/Surveillance Task Force}

Adebola Adebayo, National Center for Immunization and Respiratory Diseases, CDC; Jennifer Adjemian, Center for Surveillance, Epidemiology, and Laboratory Services, CDC; Minal Amin, National Center for Immunization and Respiratory Diseases, CDC; Jose Aponte, Center for Surveillance, Epidemiology, and Laboratory Services, CDC; Grace Appiah, National Center for Emerging and Zoonotic Infectious Diseases, CDC; Christina Armatas, California Department of Public Health; Melissa Arons, Epidemic Intelligence Service, Center for Global Health, CDC; Sukhshant Kaur Atti, Agency for Toxic Substances and Disease Registry, CDC; Michelle Banks, National Center for Immunization and Respiratory Diseases, CDC; Vaughn Barry, Epidemic Intelligence Service, National Center for Injury Prevention and Control, CDC; Amy Board, Epidemic Intelligence Service, National Center for Injury Prevention and Control, CDC; Tegan Boehmer, National Center for Environmental Health, CDC; John Bowyer, National Center for Emerging and Zoonotic Infectious Diseases, CDC; Lauren BoyleEstheimer, National Center for Chronic Disease Prevention and Health Promotion, CDC; Diane Browning, Northrop Grumman; David Bui, Epidemic Intelligence Service, National Center for Environmental, CDC; Jordan Cates, Epidemic Intelligence Service, National Center for Immunization and Respiratory Diseases, CDC; Gyan Chandra, National Center for Chronic Disease Prevention and Health Promotion, CDC; Karen Chang, Epidemic Intelligence Service, National Center for Chronic Disease Prevention and Health Promotion, CDC; Jennifer Chevinsky, Epidemic Intelligence Service, National Center for Chronic Disease Prevention and Health Promotion, CDC; Katelyn Chiang, National Center for Chronic Disease Prevention and Health Promotion, CDC; Pyone Cho, National Center for Chronic Disease Prevention and Health Promotion, CDC; George Cone, National Center for Immunization and Respiratory Diseases, CDC; Matthew Cone, Council of State and Territorial Epidemiologists; Kristen Cowan, National Center for Environmental Health, CDC; Augustina Delaney, G2S Corporation; Lindsey Duca, Epidemic Intelligence Service, National Center for Chronic Disease Prevention and Health Promotion, CDC; Angela Dunn, Utah Department of Health; Shideh Delrahim Ebrahim-Zadeh, National Center for Environmental Health, CDC; Jeff Engel, Council of State and Territorial Epidemiologists; Molly Evans, National Center for Injury Prevention and Control, CDC; Victoria Fields, Epidemic Intelligence Service, National Center on Birth Defects and Developmental Disabilities CDC; Aaron Fleishauer, North Carolina Department 
of Health and Human Services; Jennifer Freed, Agency for Toxic Substances and Disease Registry, CDC; Allison Gately, National Center for Injury Prevention and Control, CDC; Isaac Ghinai, Illinois Department of Health; Caitlin Green, National Center on Birth Defects and Developmental Disabilities, CDC; Janet Hamilton, Council of State and Territorial Epidemiologists; Arianna Hanchey, National Center for Environmental Health, CDC; Kathleen Hartnett, Center for Surveillance, Epidemiology, and Laboratory Services, CDC; Amy Heinzerling, California Department of Public Health; Dessica Hodges, Mercer University; Brooke Hoots, National Center for Injury Prevention and Control, CDC; Asad Islam, Center for Surveillance, Epidemiology, and Laboratory Services, CDC; Mia Israel, Council of State and Territorial Epidemiologists; Yunho Jang, National Center for Immunization and Respiratory Diseases, CDC; Sumera Jiva, Mercer University; Jona Johnson, Agency for Toxic Substances and Disease Registry, CDC; Charlotte Kaboré, National Center for Chronic Disease Prevention and Health Promotion, CDC; Emily Kiernan, Office of the Director, CDC; Uzay Kirbiyik, Center for Surveillance, Epidemiology, and Laboratory Services, CDC; Hannah Kisselburgh, National Center for Emerging and Zoonotic Infectious Diseases, CDC; Mimisha Kothari, Center for Surveillance, Epidemiology, and Laboratory Services, CDC; Vikram Krishnasamy, National Center for Injury Prevention and Control, CDC; Mohammed Lamtahri, Center for Surveillance, Epidemiology, and Laboratory Services, CDC; Michael Landen, New Mexico Department of Health; Jennifer Layden, Illinois Department of Public Health; Mark Layer, National Center for Environmental Health, CDC; Ruth Lynfield, Minnesota Department of Health; Kristen Marshall, Epidemic Intelligence Service, Center for Surveillance, Epidemiology, and Laboratory Services, CDC; Keegan McCaffrey, Utah Department of Health; Eva McLanahan, Office of the Director, CDC; Jonathan Meiman, Wisconsin Department of Health; Christina Mikosz, National Center for Injury Prevention and Control, CDC; Maureen Miller, Epidemic Intelligence Service, National Center for Chronic Disease Prevention and Health Promotion, CDC; Roger Mir, Center for Surveillance, Epidemiology, and Laboratory Services, CDC; Yousra Mohamoud, National Center for Chronic Disease Prevention and Health Promotion, CDC; Livia Navon, Illinois Department of Public Health; Varsha Neelam, National Center on Birth Defects and Developmental Disabilities, CDC; David Nitschke, Center for Surveillance, Epidemiology, and Laboratory Services, CDC; Rashid Njai, Office of the Deputy Director for Non-Infectious Diseases, Office of the Director, CDC; Kevin O'Laughlin, Epidemic Intelligence Service, National Center for Emerging and Zoonotic Infectious Diseases, CDC; Samantha Olson, G2S Corporation; Cria Perrine, National Center for Chronic Disease Prevention and Health Promotion, CDC; Cassandra Pickens, National Center for Injury Prevention and Control, CDC; Mary Pomeroy, Epidemic Intelligence Service, National Center for Emerging and Zoonotic Infectious Diseases, CDC; Aaron Kite Powell, Center for Surveillance, Epidemiology, and Laboratory Services, CDC; Ian Pray, Wisconsin Department of Health Services; Tia Rogers, Epidemic Intelligence Service, National Center for Injury Prevention and Control, CDC; Nicole Roth, Eagle Medical Services; Phillip Salvatore, Epidemic Intelligence Service, National Center for Injury Prevention and Control, CDC; Caroline Schrodt, Epidemic Intelligence Service, National Center for Emerging and Zoonotic Infectious Diseases, CDC; Sarah Shafer, National Center for Immunization and Respiratory Diseases, CDC; Dhara Shah, Council of State and Territorial Epidemiologists; Raschelle Smalley, Mercer University; Steven Sumner, National Center for Injury Prevention and Control, CDC; Lauren Tanz, North Carolina Department of Health and Human Services; Mark Tenforde, Center for Surveillance, Epidemiology, and Laboratory Services, CDC; Vilma Thomas, National Center for Chronic Disease Prevention and Health Promotion, CDC; Megan Toe, Council of State and Territorial Epidemiologists; Kate Varela, Epidemic Intelligence Service, National Institute for Occupational Safety and Health; Alana Vivolo, National Center for Injury Prevention and Control, CDC; Jason Wilken, California Department of Public Health; Peter Yang, National Center for Chronic Disease Prevention and Health Promotion, CDC; Anna Yousaf, National Center for Immunization and Respiratory Diseases, CDC.

\section{References}

1. Siegel DA, Jatlaoui TC, Koumans EH, et al.; Lung Injury Response Clinical Working Group; Lung Injury Response Epidemiology/ Surveillance Group. Update: interim guidance for health care providers evaluating and caring for patients with suspected e-cigarette, or vaping, product use associated lung injury-United States, October 2019. MMWR Morb Mortal Wkly Rep 2019;68:919-27. https://doi. org/10.15585/mmwr.mm6841e3

2. Perrine CG, Pickens CM, Boehmer TK, et al.; Lung Injury Response Epidemiology/Surveillance Group. Characteristics of a multistate outbreak of lung injury associated with e-cigarette use, or vaping-United States, 2019. MMWR Morb Mortal Wkly Rep 2019;68:860-4. https://doi. org/10.15585/mmwr.mm6839e1
3. Ghinai I, Pray IW, Navon L, et al. E-cigarette product use, or vaping, among persons with associated lung injury-Illinois and Wisconsin, April-September 2019. MMWR Morb Mortal Wkly Rep 2019;68:865-9. https://doi.org/10.15585/mmwr.mm6839e2

4. Lewis N, McCaffrey K, Sage K, et al. E-cigarette use, or vaping, practices and characteristics among persons with associated lung injury-Utah, April-October 2019. MMWR Morb Mortal Wkly Rep 2019;68:953-6. https://doi.org/10.15585/mmwr.mm6842e1

5. CDC. Outbreak of lung injury associated with e-cigarette use, or vaping. Atlanta, GA: US Department of Health and Human Services, CDC; 2019. https://www.cdc.gov/tobacco/basic_information/e-cigarettes/ severe-lung-disease.html 